Voix et Images

voixetimages

\title{
Bibliographie de Francine Noël
}

Lucie Joubert et Caroline Barrett

Volume 18, numéro 2 (53), hiver 1993

Francine Noël

URI : https://id.erudit.org/iderudit/201026ar

DOI : https://doi.org/10.7202/201026ar

Aller au sommaire du numéro

\section{Éditeur(s)}

Université du Québec à Montréal

\section{ISSN}

0318-9201 (imprimé)

1705-933X (numérique)

Découvrir la revue

\section{Citer ce document}

Joubert, L. \& Barrett, C. (1993). Bibliographie de Francine Noël. Voix et Images, 18(2), 326-332. https://doi.org/10.7202/201026ar d'utilisation que vous pouvez consulter en ligne.

https://apropos.erudit.org/fr/usagers/politique-dutilisation/ 


\section{Bibliographie de Francine Noël}

\section{Lucie Joubert, Université McGill,}

avec la collaboration de Caroline Barrett, Université Queen's

\section{GEUVRES \\ I.1. Volumes}

Maryse, roman, Montréal, VLB éditeur, 1983, 426 p.

Maryse, roman, préface de Lise Gauvin, Montréal, VLB éditeur, coll. *Courant , 1987, $444 \mathrm{p}$.

Chandeleur. Cantate parlée pour cinq voix et un mort, thêâtre, Montréal, VLB éditeur, 1985, $187 \mathrm{p}$.

Myriam première, roman, Montréal, VLB éditeur, 1987, 532 p.

Babel, prise deux ou Nous avons tous découvert l'Amérique, Montréal, VLB éditeur, 1990, $411 \mathrm{p}$.

Nous avons tous découvert l'Amérique, roman, Montréal/Arles, Leméac/Actes Sud, 1992, 323 p.

1.2. Article critique, préface et commentaire

-Les bonnes intentions suffisent-elles?,, Jeu. Cabiers de thêâtre, n 19, 1981.2, p. 170-171.

- Plaidoyer pour mon image ", Jeu. Cabiers de théâtre, $n^{\circ} 16,1980.3$, p. 23-56.

-Thérèse et Pierrette, et Simone, sont-elles "regardables"? ", introduction à Michel Tremblay, Thérèse et Pierrette à l'École des Saints-Anges, Montréal, Bibliothèque québécoise, 1991, p. 7-13.

\subsection{Mémoire et Thèse}

- L'esthétique du conte chez Charles Perrault , mémoire présenté à la Faculté des lettres de l'Université de Montréal, pour l'obtention du Diplôme d'études supérieures, Montréal, Université de Montréal, 1970, $120 \mathrm{f}$.

- Du sujet noyé: l'innommable de Samuel Beckett *, thèse de doctorat, Université de Vincennes, Paris XIII, 1972, $300 \mathrm{f}$.

\section{I.4. En collaboration}

- Les colonies, l'exotisme et la note au bas de la page., Lise Gauvin et Jean-Marie Klinkenberg (directeurs), Ecrivain cherche lecteur. L'écrivain francophone et 
ses publics, Montréal/Paris, VLB éditeur/Créaphis, coll. -Rencontres à Royaumont *, 1991, p. 51-62.

-[Sans titre], Marie-Christine Abel (directrice), Montréal en mots, Montréal en couleurs, Montréal, Stanké/Beauchamp/Savoie, 1991, p. 177-186.

- La scène se passe à Montréal, de nos jours ;, Lire Montréal. Actes du Colloque tenu le 21 octobre 1988 onganisé par le groupe de recherche .Montréal imaginaire, Département d'études françaises, Université de Montréal, 1989, p. 120-134.

\section{ENTREVUES}

DE GRAMONT, Monique, -Maryse, Sarah et les autres: entrevue avec Francine Noël „, Châtelaine, vol. XXVI, n 12, 25 décembre 1985, p. 28-30, 32.

FORTIN, André, - Dialogues avec Francine Noël et Michel Tremblay", Recherches sociographiques, vol. XXXI, n 1, janvier-avril 1990, p. 73-84.

GAUVIN, Lise, -À propos de langue et d'écriture. Cèla rejoint la poésie •, Possibles, vol. XI, n³, printemps-été 1988, p. 208-210.

LACHANCE, Micheline et Monique ROY, :Scarlett ", Châtelaine, vol. XXXII, $n^{\circ} 8$, août 1991, p. 18-22.

MARTEL, Réginald, -Je me sens romancière américaine: entrevue avec Francine Noël , La Presse, 4 février 1984, p. B-1-B-2.

NORMAND, Anne, Ia triple nature de Francine Noël: entrevue avec Francine Noël , La Presse, 26 août 1985, p. A-8.

PARE, Isabelle, :Mai 1968: qu'en reste-t-il ? , Châtelaine, vol. XXIX, n 5, mai 1988, p. 48-56.

ROY, Monique, "Et Maryse vint: entrevue avec Francine Noël „, Le Devoir, 4 février 1984, p.17, 22.

TREPANIER, Marie-Claude, -Francine Noël. Une vieille passion *, La Vie en rose, n 32, décembre-janvier 1986, p. 48-49.

TRUDEL, Serge, -Le livre du mois. Maryse. [Entrevue],, Nos livres, vol. XV, mars 1984, p. 4-5.

VIAU, Robert, -Montréal, Myriam, Maryse: entrevue avec Francine Noël ", Lettres québécoises, n 57 , printemps 90 , p. 16-19.

\section{III. ÉTUDES}

III.1. Articles de périodiques

III.1.1. Articles de périodiques sur Maryse

[ANONYME], - Maryse`, Québec Hebdo, vol. VI, n 8, 5 mars 1984, p. 4.

[ANONYME], - Maryse, de Francine Noël., Revue du Réseau d'action et d'information pour les femmes, $\mathrm{n}^{\text {o* }} 85-86$, juillet-août 1984, p. 73-79.

[ANONYME] • Maryse., Booklist, vol. LXXXVII, 15 janvier 1991, p. 1045.

BARRETT, Caroline, *Maryses, Québec français, n 53, mars 1984, p. 8-9.

BEAULIEU, Michel, -Maryse *, Livres d'ici, vol. IX, n 5, 1984, p. 9. 
BOIVIN, Jean-Roch, .Ecrivains nouvelle vague ou nouvelle vague d'écrivains *, Montréal ce mois-ci, février 1984, p. 18.

CLICHE, Anne Élaine, - Paradigme, palimpseste, pastiche, parodie dans Maryse de Francine Noël ., Voix et Images, vol. XII, n 3, prntemps, 1987, p. 430-438.

CLOUTIER, Guy, -Francine Noël: Jésus s'appelait Myriam et sa gardienne écrivait dés poèmes *, Nuit blanche, $n^{\circ} 18$, avril-mai 1985, p. $40-43$.

CORRIVAULT, Martine R., -Beau roman pour bohème nostalgique ,, Le Soleil, 15 décembre 1983, p. D-12.

DUBOIS, Richard, *Chut! Voici Maryse... Relations, vol. XIIV, n 500, mai 1984, p. 140.

DUFRESNE, Mario, •Le passé immédiat éclaire-t-il le futur? •, Hebdo St-Louis, vol. I, $n^{\circ} 24,18$ avril 1984, p. 12.

FOGLIA, Pierre, •Quelques titres contre la grippe „, La Presse, 29 décembre 1983, p. C-9.

GAUVIN, Lise, -Maryse, le livre de ceux qui avaient 20 ans en 68, Le Devoir, 21 janvier 1984, p. 15.

- - Montréal est une fête", Possibles, vol. VIII, no 3, p. 41-53. [repris dans Lettres d'une autre, Montréal / Paris, l'Hexagone/Le Castor astral, 1984, p. 82-84.]

GILBERT, Bernard, ·[Maryse] ", Intervention, n²2-23, 1984, p. 95.

HOMEL, David, -Maryse», Books in Canada, vol. XIV, n' 1, janvier-février 1985, p. 2-3.

LEFEBVRE, Pierre, *Maryse *, Mcebius, n² 20, hiver 1984, p. 67-68.

MAILJÉ, Michèle, •Des livres agréables pour Pâques ", Le Nouvelliste, 17 avril 1984.

MARCHESSAULT, Jovette, "La débarque des "paètes" ", La Vie en rose, $n^{\circ} 16$, mars 1984, p. 59.

MARCOTTE, Gilles, •Maryse. Tendrement satirique, le roman des années "flyées" ", L'Actualité, vol. IX, n 4, avril 1984, p. 113.

—, "La poésie Oubedon ", Úrgences, $n^{\circ} 28$, mai 1990, p. 68-78.

MARTEL, Réginald, • Littérature ", La Presse, 23 juin 1984, p. D-3. p. C-3.

MICHON, Jacques, "[Maryse]•, University of Toronto Quarterly, vol. LIII, n 4, été 1984, p. 341-342.

PELLETIER, Jacques, - Maryse ou le portrait d'une génération •, Pour le socialisme, $\mathrm{n}^{\circ}$ 5, deuxième semestre, 1984, p. 18.

ROY, Michelle, “Écrire par amour ou par ambition?`, La Vie en rose, $\mathrm{n}^{\circ} 19$, septembre 1984, p. 50.

SAINT-MARTIN, Lori, “Maryse, de Francine Noël ", Resources for Feminist Research/ Documentation sur la recherche féministe, vol. XIII, $\mathrm{n}^{\circ} 3$, novembre 1984 , p. 57-58. 
STANTON, Julie, -Avoir 20 ans en 68", La Gazette des femmes, vol. VI, n 3, septembre-octobre 1984, p. 4.

THÉORET, France, : Grandeur et misère du roman •, Spirale, n 41, mars 1984, p. 3. TREPANIER, Marie-Claude, - Maryse ", Nuit blanche, n 12, février-mars 1984, p. 18.

TRUDEL, Danielle, - Maryse ou c'était avant le déclin de l'empire américain de Francine Noël •, Québec français, n 68, décembre 1987, p. 85-86.

TRUDEL, Serge, *Maryse", Nos livres, vol. XV, mars 1984, p. 31-33.

VANASSE, André, .Papa Tom, Elisa Doolitle et les autres", Lettres québécoises, $n^{\circ} 33$, printemps 1984, p. $34-35$.

\section{III.1.2. Articles de périodiques sur Chandeleur}

[ANONYME], "Chandeleur: cantate parlée pour 5 voix et un mort. Compte rendu *, Canadian Woman Studies, vol. VIII, n 1, printemps 1987, p. 114.

ACKERMAN, Marianne, -Novelist's First Foray Into Theater Produces a Solid, Original Work: Theater Review on Chandeleur, The Gazette, 29 janvier 1986, p. E-7.

ANDRÈ, Bernard, “Viser/rater la cible•, Spirale, n 59, mars 1986, p. 8-9.

BERNATCHEZ, Raymond, "Un passage au théâtre: Francine Noël •, La Presse, 18 janvier 1986, p. E-5.

DIONNE, André, -Le thêâtre qu’on joue», Lettres québécoises, n 42, été 1986, p. 49-51.

ROBERT, Lucie, -Dramaturgie: la littérature au théâtre „, Voix et Images, vol. XII, $\mathrm{n}^{\circ} 1$, automne 1986 , p. $155-160$.

VAIS, Michel, *Traces ", Jeu. Cabiers de théâtre, n 39, 1986, p. 155-156.

\section{III.1.3. Articles de périodiques sur Myriam première}

BARBE, Jean, •Francine Noël. L'école des femmes •, Voir, 17 au 23 décembre 1987, p. 6.

BISSONNETTE, Lise, "Writer Celebrates "That Bit of Happiness" "Globe and Mail, 13 août 1988 , p. D-2.

BOIVIN, Jean-Roch, * Pour l'amour des grands-mères, dés petites filles et des petits garçons, de l'art, de Montréal et pour l'amour tout court., Le Devoir, 12 décembre 1987, p. D-3.

DROUIN, Serge, - Myriam première. Des réflexions sur le monde adulte et celui de l'enfance ", Journal de Québec, 23 janvier 1988, p. 9-A.

DUBOIS, Richard, -Lecture. Recensions de mai. Myriam première ", Relations, $n^{\circ}$ 540, mai 1988, p. 124-126.

HÉBERT, Pierre, • Les plaisirs de lire ", Voix et Images, vol. XIV, n 1, automne 1988, p. $130-135$. 
LAFOREST, Marty, - Myriam première *, Nuit blanche, n 32 , juin-juillet 1988, p.1516.

LAURIER, Marie, -Myriam première. Le deuxième roman de Francine Noël ", Le Devoir, 18 novembre 1987, p. 11.

LAURIN, Michel, Myriam première. Roman de Francine Noël", Nos livres, vol. $\mathrm{XIX}, \mathrm{n}^{\circ}$ 3, avril 1988, p. 23-24.

MAILLE, Michèle, : Myriam première, le début d'un nouveau monde? ", Le Journal de St-Bruno, 12 janvier 1988, p. 19.

MARCOTTE, Gilles, -Familles, je vous haime ", L'Actualité, vol. XIII, n 3, mars 1988, p. 149.

MARTEL, Réginald, : Myriam première. Francine Noël signe un des trois ou quatre grands romans de la décennie ", La Presse, 19 décembre 1987, p. J-1-J-2.

MILOT, Louise, •Francine Noël à l'école de Michel Tremblay •, Lettres québécoises, n' 50, été 1988, p. 22-23.

MONTESSUIT, Carmen, -Après Maryse, Francine Noël récidive avec Myriam première, Le Journal de Montréal, 23 novembre 1987, p. 40.

PELLETIER, Francine, *Fairy tale for the '80s *, The Gazette, 5 mars 1988, p. J-1.

PELLETIER, Marie-Ève, :Myriam, première [sic] de F. Noël. Le tableau d'un temps bien vécu mais résolu ", Le Droit, 30 janvier 1988, p. 50.

ROY, Monique, - Francine Noël de Myriam première: "J'ai voulu montrer l'importance de la transmission d'une culture" ", La Presse, 27 février 1988, p. J-1$\mathrm{J}-2$.

ROYER, Jean, " J'écris sur Montréal car toute littérature se doit d'être incarnée" Francine Noël •, Le Devoir, 12 mars 1988, p. D-1.

SIMARD, Mireille, -Myriam première *, Châtelaine, vol. XXIX, $\mathrm{n}^{\circ} 3$, mars 1988, p. 25.

SIMON, Sherry, •Du plateau à Griffintown •, Spirale, n 77, mars 1988, p. 12.

TROTTIER, Barbara, : La ruelle Mentana: tout un monde !*, Ecrits du Canada français, $n^{\circ} 63,1988$, p. 183-187.

VOISARD, Anne-Marie, .Francine Noël, "fignoleuse" de l'écriture», Le Soleil, 23 janvier 1988, p. D-1-D-2.

Myriam première de Francine Noël. Un roman dont l'hérö̈ne restera gravée dans nos mémoires ", Le Soleil, 9 janvier 1988, p. C-8.

III.1.4. Articles de périodiques sur Babel, prise 2 ou Nous avons tous découvert l'Amérique

[ANONYME] "Babel, prise deux ou Nous avons tous découvert l'Amérique", Booklist, vol. LXXXVIII, 1 ${ }^{\text {er }}$ février 1992, p. 1013.

BASILE, Jean, - Francine Noël et Hélène Rioux: de vrai bavardage en faux [sic] atmosphère :, Le Devoir, 10 novembre 1990, p. D-7. 
BERTIN, Raymond, Mosaïque des doutes et des désirs", Guide Mont-Royal, 28 novembre 1990, p. 14.

BOIVIN, Jean-Roch, :La planète des signes *, Voir, 8 au 14 novembre 1990, p. 26.

CHAMPAGNE, Christine, :Des cahiers comme preuve d'existence ", Lettres québécoises, n 61, printemps 1991, p. 21-22.

CÔTE, Lucie, :Le Montréal Babel de Francine Noël •, La Presse, 4 novembre 1990, p. C-5.

FOGLIA, Pierre, "Lire, mon vieux •, La Presse, 13 novembre 1990, p. A-5.

HUDON, Jean-Guy, - Nouveautés ", Québec français, n 81, printemps 1991, p. 18.

JARQUE, Alexandra, *Babel, prise deux*, Nuit blanche, n 43, mars-avril-mai 1991, p. 19-20.

LAURIN, Lucie, -Lucidité et fantaisie., Nouvelles CSN, n³16, 30 novembre 1990, p. 21.

MARCOTTE, Gilles, •Fatima Gagné, orthophoniste „, L'Actualité, vol. XVI, n.3, 1 mars 1991, p. 71.

MARTEL, Réginald, - La Mauvaise Foi, de Tougas, un titre marquant en 1990 • La Presse, 30 décembre 1990, p. C-2.

MONTESSUIT, Carmen, "Babel, prise deux: plus un journal intime qu'un roman ", Le Journal de Montréal, 15 décembre 1990, p. WE-15.

PELLETIER, Marie-Ève, - Un monde où tous pourront se trouver *, Le Droit, 3 novembre 1990 , p. A-6.

PICARD, Geneviève, * Lectures •, Elle-Québec, n. 17, janvier 1991, p. 40.

ROY, Monique, . Francine Noël et sa tour de Babel •, Châtelaine, vol. XXXI; $\mathbf{n}^{\circ} 10$, octobre 1990, p. 30.

SIMON, Sherry, :Tours de Babel ", Spirale, n 104, mars 1991, p. 3.

VOISARD, Anne-Marie, - Le dernier roman de Francine Noël: thèmes sérieux et actuels sur un mode drôle et entraînant , Le Soleil, 3 novembre 1990, p. E-13.

\section{III.1.5. Articles de périodiques généraux}

BISHOP, Neil B., - Le personnage français dans quelques romans québécois contemporains, Voix et Images, vol. XIII, n 1, automne 1987, p. 82-103.

COLVILE, Georgiana, -L'univers de Francine Noël *, Québec Studies, n 10, 1990, p. 99-105.

DEMERS, Dominique, *Les tricoteuses de saga ", L'Actualité, vol. XIII, $\mathrm{n}^{\circ} 7$, juillet 1988, p. 91-94.

JARQUE, Alexandra, - Le roman des copines ", Possibles, vol.15, n², printemps 1991, p. 31-42.

MICHAUD, Ginette, : Mille plateaux: topographie et typographie d'un quartier ", Voix et Images, vol. XIV, n³, printemps 1989, p. 462-482. 
SAINT-MARTIN, Lori, "Le métaféminisme et la nouvelle prose féminine au Québec ", Voix et Images, vol. XVIII, n 1, automne 1992, p. 78-88.

\section{III.2. Mémoire et chapitres de volumes}

BENEDICT, Francesca D., - Discours de femmes et discours sur la femme dans deux romans québécois contemporains: Maryse et La grosse femme d'à côté est enceinte, mémoire de maîtrise en Études littéraires, Montréal, Université du Québec à Montréal, 1987, 112 f.

GAUVIN, Lise et Gaston MIRON, "Francine Noël", Ecrivains contemporains du Québec, Paris, Seghers, 1989, p. 411-414 [Extraits de Maryse et de Myriam première].

GAUVIN, Lise, et Franca MARCATO-FALZONI (directrices), L'Âge de la prose. Romans et Récits québécois des années 80, Montréal/Rome, VLB éditeur/ Bulzoni Editore, Montréal, 1992, passim.

LARUE, Monique, en collaboration avec Jean-François CHASSAY, Promenades littéraires dans Montréal. Photos originales de Yvan Dubé, Montréal, Éditions Québec/Amérique, 1989, passim.

SAINT-MARTIN, Lori, - Writing (Jumping) off the Edge of the World: Metafeminism and New Women Writers of Québec:, Karen Gould (directrice), Beyond the Hexagon, à paraître.

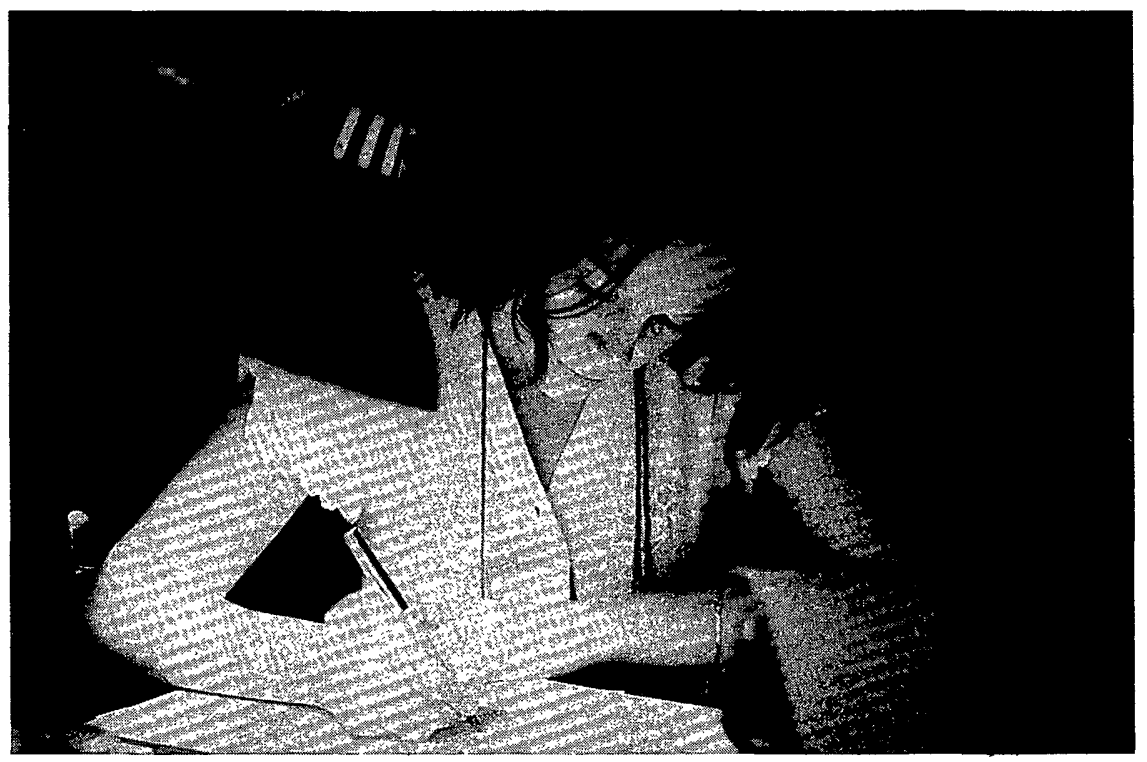

Photo: André A. Bélanger 